\title{
Evaluation of the Efficacy of a New Intensive Antiaging Treatment Based on the Combination of the Secretion of Cryptomphalus aspersa, Vitamin $\mathrm{C}$ and Proteoglycans
}

\author{
M Vitale ${ }^{1}$, A Perez-Davo ${ }^{1}$, C Zhihao $^{2}$, V Nobile $^{3}$ and MT Truchuelo ${ }^{4 *}$ \\ ${ }^{1}$ Medical affairs department, Cantabria labs, Madrid, Spain \\ ${ }^{2}$ Complife Group, China \\ ${ }^{3}$ Complife Group, Milán, Italy \\ ${ }^{4}$ Dermatology Department, Vithas Nuestra Señora de America Hospital, Madrid, Spain
}

*Corresponding author: MT Truchuelo, Dermatology Department, Vithas Nuestra Señora de America Hospital, Madrid, Spain, Tel: 34606516077 ; E-mail: maytetd@yahoo.es

Received: 23 Oct, 2017 | Accepted: 10 Jan, 2018 | Published: 16 Jan, 2018

Citation: Vitale M, Perez-Davo A, Zhihao C, Nobile V, Truchuelo MT (2018) Evaluation of the Efficacy of a New Intensive Antiaging Treatment Based on the Combination of the Secretion of Cryptomphalus Aspersa, Vitamin C and Proteoglycans. J Clin Cosmet Dermatol 2(1): dx.doi. org/10.16966/2576-2826.123

Copyright: (c) 2018 Vitale $M$, et al. This is an open-access article distributed under the terms of the Creative Commons Attribution License, which permits unrestricted use, distribution, and reproduction in any medium, provided the original author and source are credited.

\section{Abstract}

Photoaging is the result of the combination of chronologic aging and the exposition to several extrinsic agents that trigger oxidative stress. It is characterized by dermal and epidermal alterations, such as wrinkles, dryness, sagging, roughness and pigmentary changes. Some of these signs are the result of an impairment of the Extracellular Matrix (ECM) components, which represent the structural and functional dermal support in healthy skin, but are diminished or dysfunctional in aged skin.

The aim of the study was to determine the antiaging effects of a new cosmetic treatment formulated with a combination of the Secretion of Cryptomphalus aspersa (SCA), Vitamin C and proteoglycans. 20 Caucasian and 20 Asian female subjects with mild to moderate photoaging were included in the study, and applied the cosmetic product twice daily on the face for 30 days. To assess its antiaging efficacy, clinical evaluation and instrumental determinations were performed at baseline, 15 days and 30 days. Skin moisturization, skin brightness, skin firmness and elasticity, and wrinkle depth were evaluated.

The results showed a significant improvement of all these parameters as soon as 15 days after starting the treatment, which was maintained or, in some cases, increased at the end of the study period. In addition, excellent tolerance was referred by all the patients. Due to the fast effects achieved with this new cosmeceutical product for the global improvement of the main signs of photoaging, it could be indicated as an intensive treatment of this condition.

Keywords: Cutaneous regeneration; Antiaging; Cellular Proliferation; Dermal regeneration; Vitamin C; Proteoglycans; Cryptomphalus aspersa; Brightening

\section{Introduction}

Global aging is a somatic process entailing the progressive loss of physiological functions, metabolic efficiency, stress resistance, and adaptive potential, and it's manifested as intrinsic or chronologic aging. The skin with intrinsic aging is defined as a degenerative process due to declining physiological functions and capacities (genetic, somatic capacity and composition). The skin is the primary organ through which the human body interacts with its environment and it is exposed to different physical, chemical and mechanical factors which may deteriorate the skin and induce the extrinsic aging, also called photoaging. These are a distinctive deteriorating process caused by environmental agents, such as solar radiation, pollution, toxic products, and life style, including nutrition and stress [1].

Photoaging involves a prominent cutaneous transformation clinically characterized by sallowness, blotchy pigmentation, increased fragility, and rough texture of the epidermis, while coarse wrinkles, sagging and telangiectasia appear due to aged structures in the dermis.

Histological and ultra structural studies have revealed that during aging, the epidermis loses its ability to eliminate extrinsic macromolecular damage compromising the integrity of the skin [2]. Additionally, an imbalance in the equilibrium between the accumulation and the degradation of Extracellular Matrix (ECM) components triggers dermal damage, consisting of impaired and disorganized collagen type I fibrils and substantial accumulation of abnormal elastotic material $[3,4]$. Thus, as the skin's tensile strength and mechanical properties rely on the ECM, its deterioration is essentially responsible for wrinkling and sagging [5]. The search for safe and noninvasive treatments to reverse the skin aging signs remains challenging.

There are two main groups of agents usually employed as anti-aging actives: the antioxidants and the cell regulators. The 
antioxidants (vitamins, polyphenols and flavonoids) reduce collagen degradation by reducing the concentration of reactive oxygen species in the tissues. The cell regulators (retinoids, peptides and growth factors) have direct effects on collagen metabolism and influence collagen production [6]. A new product with the combination of actives from these two groups was developed.

One of the three main ingredients of the new product, the secretion of the mollusk Cryptomphalus aspersa (SCA) is a registered active (IF Cantabria labs, SA) with proclaimed antiaging effects. Cryptomphalus aspersa is a gastropod from the Helicidae family whose secretion (SCA) is obtained through a patented process where the snails are stimulated and their secretions are filtered and then collected and tested for purity and activity. It is important highlight that snails are not harmed during this process.

The SCA is rich in glycosaminoglycans and Growth Factors (GFs) that induce skin regeneration by stimulating biochemical, structural and functional processes of the skin $[7,8]$. SCA is an ingredient with antioxidant activity that increases the physiology, proliferation and migration of fibroblasts and keratinocytes, improving the cell structure and promoting the secretion and assembly of ECM $[9,10]$, thus helping to prevent elastosis in photo damaged skin. SCA has also demonstrated to accelerate wound healing [11].

Clinical assessment of the effects of SCA has showed that topical treatment with SCA reverse some of the features of photoaging, increasing skin thickness and reducing roughness and wrinkle depth, likely due to its antioxidant and matrixremodeling effects [12]. On one of the last studies published, (a multicenter, double-blind and randomized study), 25 patients with moderate to severe facial photodamage were treated with SCA on one side of the face and placebo on the contralateral side for 12 weeks. The results demonstrated that periocular rhytides on the active ingredient side showed significant improvement after 12 weeks $(\mathrm{P}=0.03)$ and improved texture to a greater degree than placebo at 8 and 12 weeks. The authors concluded that daily application of products containing SCA proved to be effective and well tolerated for improvement in coarse periocular rhytides and fine facial rhytides [13].

With the idea to obtain a new cosmeceutical product with regenerative properties and enhancing the skin brightness and moisturization; SCA (dermal regeneration) was combined with two interesting and well known ingredients: Vitamin $\mathrm{C}$ (brightness) and proteoglycans (moisturization) (Endocare ${ }^{\varpi} \mathrm{C}$ Pure concentrate oil free).

Proteoglycans (PGs) are composed by glycosaminoglycans and/or oligosaccharides attached to a core protein. PGs can interact with other ECM components to conform and stabilize the ECM, thus promoting hydration and increasing the permeability of low molecular weight solutes [14]. Three major PGs are present in human skin: versican, decorin and a catabolic fragment of decorin, termed decorunt [15]. mRNA levels of several PGs have been shown to be down regulated by UV irradiation [16], which may have weakening effects on dermal matrix structures with down regulation of procollagen I by UV [17-19].

Vitamin C (L-ascorbic acid) has an important effect on hyper pigmentation [20]. It is a potent antioxidant owing to its ability to scavenge Reactive Oxygen Species (ROS). This antioxidant effect prevents melanogenesis and positions Vitamin $\mathrm{C}$ as a defense against oxidative radicals and a useful agent for treating photoaging and other dermatologic conditions [21]. The brightening and lightening effect induced by Vitamin $\mathrm{C}$ is also mediate by the inhibition on the activity of tyrosinase through its antioxidant effect [22] or via its ability to reduce the enzymatically generated $o$-quinones, thus reducing melanin synthesis [23]. It also minimizes oxidized melanin, changing the pigmentation from black to tan. Topical application of Vitamin $\mathrm{C}$ has demonstrated to be photo protective and prevent some of the actinic damage [24-26]. By preventing the absorption of ultraviolet radiation (both UVA and UVB, which trigger melanogenesis) also induces skin brightening [27]. In addition, this vitamin is necessary for the synthesis of another antioxidant vitamin, i.e., Vitamin E. Furthermore, Vitamin C is able to improve wrinkling and atrophy of the aged skin $[28,29]$ due to its capacity to suppress Matrix-Metalloproteinases (MMPs), responsible for collagen degradation, increase the Tissue Inhibitor of MMPs (TIMPs) and the levels of collagens I and III [30,31]. The new product contains high and stabilized Vitamin C concentrations.

With the new cosmeceutical product, a clinical study was performed with the aim to demonstrate, based on non-invasive and objective evaluation techniques, if these combinations could improve different skin aging parameters.

\section{Material and Methods}

\section{Study population}

The study was designed as a prospective and multicenter clinical trial where the volunteers acted as their own internal control before and after treatment. Forty volunteers were recruited (50\% were Caucasians and 50\% were Asian volunteers). The study was done by Complife Group, Italy, where the Caucasian population was recruited; and Complife Group China, where the Asian population was recruited.

The subjects participating in this study were screened and enrolled in the study under the supervision of dermatologists including healthy women with ages between $30-45$ years and in accordance with the inclusion and non-inclusion criteria.

Product efficacy was assessed after 15 and 30 days of use by means of non-invasive instrumental techniques able to measure skin moisturization (Corneometer ${ }^{\circledast}$ CM 825 (Courage+Khazaka, electronic $\mathrm{GmbH}$ ), elasticity (Cutometer ${ }^{\circledast}$ MPA 580, radiance (Colorimeter ${ }^{\circledR}$ CM-700D (Konica Minolta) and wrinkle depth (Primos 3D Lite ${ }^{\circledast}$ PRIMOS (Phase shift 
Rapid In-vivo Measurement of Skin). A self-assessment by the subjects participating in the study was carried out with a specific questionnaire.

\section{Inclusion criteria}

For the study only healthy female subjects aged between 30 and 45 years were included. Only Asian and Caucasian ethnicities were included. Phototypes of Caucasian subjects ranged from II to IV. Only those with mild to moderate photoaging clinical signs were admitted. Not to desire to get pregnant in the forthcoming months and use of contraceptives was necessary. In addition, none of the recruited subjects should have concomitant diseases nor use other topical or systemic products that could interfere with or affect the results. Those with allergy to the product ingredients were excluded.

\section{Treatment regime}

Subjects were asked to leave all other skin care products, cosmetics, nutritional supplements, and medications unchanged for the duration of the study, only adding the new intensive treatment Endocare ${ }^{\varpi}$ pure Concentrate Oil Free (Cantabria labs). The routine was base in the topical use of one daily ampule on the face, applied twice a day for 30 days. The concentration of the main ingredients content in the study's product, Endocare ${ }^{\circledast} \mathrm{C}$ Pure Concentrate Oil Free: Secretion of Cryptomphalus aspersa (SCA): 20\%; Vitamin C pure and stabilize: $15 \%$ and Proteoglycans $2 \%$. The test was conducted during autumn and early winter time.

\section{Clinical assessment}

The dermatologist investigator and the subjects completed efficacy, safety and tolerability assessments at baseline (T0), 15 days (T1) and 30 days (T2).

In addition, instrumental multi-parametric approach was measured by non-invasive bioengineering techniques: Skin moisturization (Corneometer ${ }^{\oplus}$ CM825), Skin brightness measurements (Colorimeter ${ }^{\circledR}$ CM-700D), skin elasticity and skin firmness measurements (Cutometer ${ }^{\ominus}$ MPA 580) and Wrinkle depth (Primos 3D Lite ${ }^{\circledast}$ ).

Skin moisturization (Moisture index): The used device is the Corneometer ${ }^{\circledast}$ CM 825 (Courage+Khazaka, electronic GmbH).

The Corneometer ${ }^{\varpi}$ method is based on the dielectric constant water. Corneometer ${ }^{\oplus}$ quantifies the degree of moisture content of the skin surface. An electric scatter field penetrates the first layer of the skin $(10-20 \mu \mathrm{m})$ and determines the dielectricity. It provides data on condition and type of skin as well as on the effects of drugs and cosmetics on the moisture contents of stratum corneum.

Skin brightness (Glow value): Colorimeter ${ }^{\circledR}$ CM-700D (Konica Minolta) was used as device. Spectrophotometer with vertical alignment perfectly suited for precise and repeated color measurement of shaped and curved samples. The measured parameter is the $8^{\circ}$ gloss value.
Skin elasticity and firmness: Cutometer ${ }^{\circledast}$ MPA 580 is a noninvasive suction device that measures any vertical deformation of the skin surface. It is computer controlled and automatically makes the calculations and stores the results. A negative pressure (450 mbar) is created in the device and the skin was drawn into the aperture of the probe for 2 seconds and after a defined time ( 2 seconds) released again. Inside the probe, the penetration depth is determinates by a non-contact optical measuring system. The resistance of the skin to the negative pressure and its ability to return into its original position are displayed as curves in real time during the measurement. R0 (skin firmness) and R2 (skin elasticity) parameters are measured.

Wrinkle depth: The used device is Primos 3D Lite ${ }^{\varpi}$. PRIMOS (Phase shift Rapid in-vivo Measurement of Skin) is an optical $3 \mathrm{D}$ in vivo skin measurement device based on digital stripe projection. It provides three-dimensional topography. The imaging system projects light on to a specific surface of the skin using a Digital Micromirror Device (DMD Texas Instruments, Irving, TX) and records the image with a CCD camera. Skin Surface microtopography is constructed using temporal phase shift algorithms to generate three-dimensional images.

Thus, subjects are asked to reply to the questions of a selfassessment questionnaire. The occurrence of any adverse event after product use was strictly followed by the dermatologist. In this case the dermatologist describes the nature, duration and the intensity of the event.

\section{Statistical analysis}

The comparison of means of outcome variables of the 5 parameters (moisture index, glow value, firmness index, elasticity index, wrinkle depth) between baseline, T15 and T30 was made by employing paired sample t-test or non-parametric Wilcoxon test, for Asian and Caucasian group, respectively. The mean differences of all the outcome measures during these comparison periods were calculated. The differences were considered to be significant at a value of $\mathrm{P}<0.05$. The null hypothesis was that the means of T0 and T15 up to T30 would be equal.

\section{Results}

40 women (20 Asians and 20 Caucasians volunteers) successfully completed the study. No tolerability issues were observed by either the dermatologist investigator or the subjects. No adverse events were reported by the patients. The largest improvement was seen in skin moisturization after 15 and 30 days of treatment, consisting of $23,5 \%$ and $26,2 \%$ improvement on Asian and Caucasian women respectively (Figure 1A).

A significant improvement of brightness in both groups was detected, as soon as for the first 15 days of treatment and even higher after one month of treatment. An improvement of $16,2 \%$ and $13,3 \%$ on Asian and Caucasian women respectively, was detected (Figure 1B). The Cutometer ${ }^{\circledast}$ showed a decrease 
of the basal maximum skin elongation both Caucasian and in Asian subjects. The decrease of the parameter correlates with an increase of firmness skin. The results have shown an improvement that was significantly achieved within the first 15 days and higher and also significant after one month of treatment. An increase of firmness of $8,1 \%$ and $9,6 \%$ on Asian and Caucasian women was observed. While the elasticity parameter correlates to an increase of the ability of the skin to return to its basal state, it showed an increase of $7,4 \%$ and $8,2 \%$, on Asian and Caucasian subjects, respectively (Figures 1C and 1D). When compared with basal level, a significant reduction of skin wrinkles was detected after 15 days of treatment and it was even more pronounced after 30 days of treatment (Figure 2).

95\% of Caucasian and $100 \%$ of Asian subjects expressed a positive opinion about the acceptability of a new cosmeceutical treatment formulated with the combination of SCA, Vitamin C and proteoglycans.

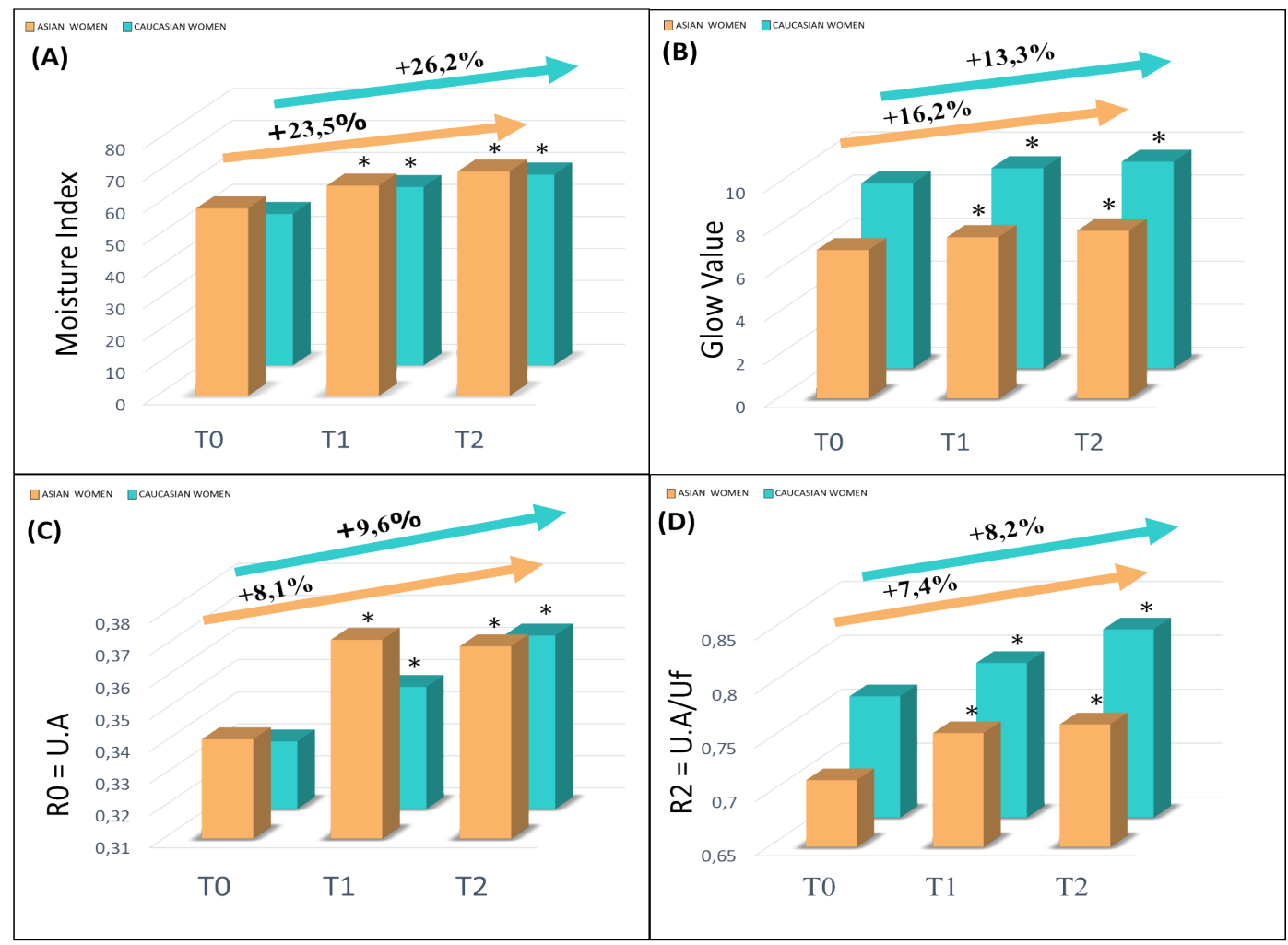

Figure 1: (A) Significant improvement of skin moisturization (Moisture index); (B) Significant improvement of skin brightness (Glow Value); (C) Significant improvement of skin Firmness (R0 parameters); and (D) Significant improvement of skin elasticity (R2 parameters) achieved at different times: T0 basal visit, T1 following 15 days and T2, after treatment (30 days). Statistical significances of the changes experienced at each time point in each group compared to baseline $\left({ }^{*} p \leq 0.001\right)$.
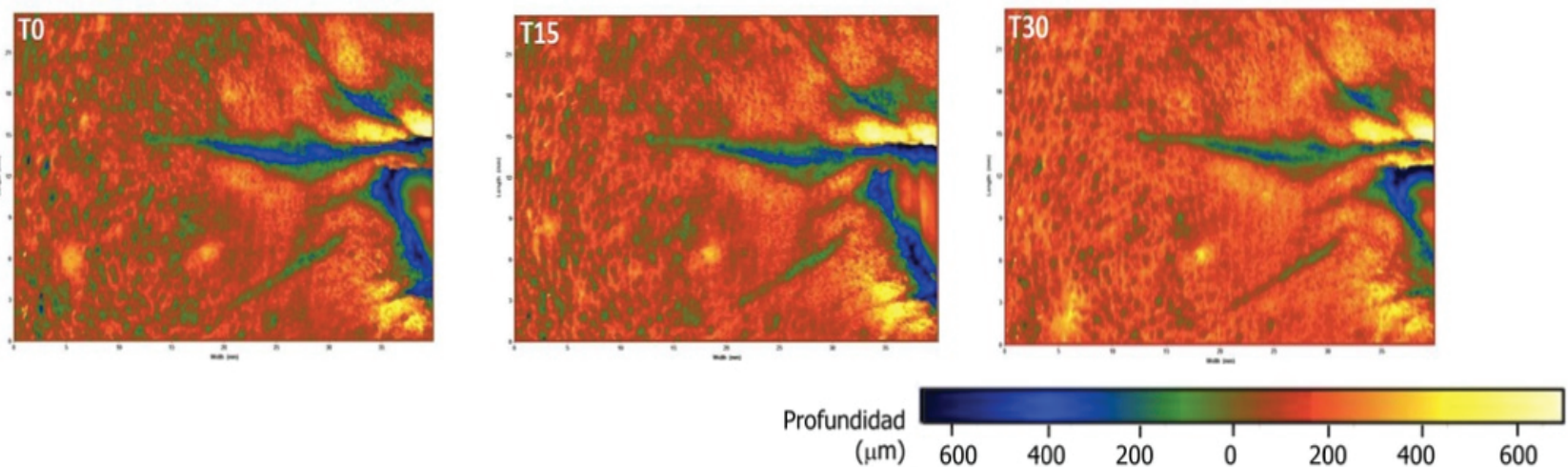

Figure 2: Significant improvement on depth wrinkles after using the cosmetic product after 15 and 30 days measured by Primos 3D Lite ${ }^{\circledR}$. The imagens belong to a Caucasian volunteer. 


\section{Discussion}

Some papers have drawn attention by reporting rejuvenating effects of Vitamin C. Fitzpatrick et al. [28] treated ten patients with a formulated Vitamin C complex having 10\% Ascorbic acid and 7\% Tetrahexyldecyl ascorbate in an Anhydrous Polysilicone gel base to one hemiface and the inactive polysilicone gel base to the other hemiface. Subjective clinical evaluation of wrinkling, pigmentation, inflammation, and hydration was performed prior to the study and at weeks 4,8 , and 12 . Two $\mathrm{mm}$ punch biopsies of the lateral cheeks were performed at 12 weeks in four patients and stained with hematoxylin and eosin, as well as in situ hybridization studies using an anti-sense probe for mRNA for type I collagen. They detected a statistically significant improvement of the Vitamin C-treated side in the decreased photoaging scores. Biopsies showed increased Grenz zone collagen, as well as increased staining for mRNA for type I collagen.

One recent and interesting paper published by Crisan et al. [30] evaluated 60 healthy women divided in three age groups (20-35, 35-50 and $>50$ years). Fifteen patients in each group were treated with a Vitamin $\mathrm{C}$ based solution and 5 patients in each age group were controls and received a placebo moisturizer cream. Evaluation was performed with high-frequency ultrasounds at basal time, 40 and 60 days after therapy. After topical Vitamin C therapy, they concluded that significantly increased of protein synthesis and collagen synthesis was obtained, related to the increase in medium and high echogenic pixels that it was shown under ultrasonographic determination. They also concluded this improvement was better in those women between 40-50 years, and decrease above 51 years [30].

In the present study we show the objective improvement of some skin aging signs when Vitamin C is topically applied in combination with proteoglycans and SCA during just one month.

The skin moisturization is very important for the perception of a healthy and attractive skin. This parameter was the one that reached the most improvement. The moisturizing effect induces soft skin and better appearance of the skin as well as a sensation of comfort perceived by the patients, and this would be of extreme importance in order to the compliance of treatment.

In our opinion, brightness could be attributed to Vitamin C effect. Studies have shown that the reduced tyrosinase activity mediated by Vitamin $\mathrm{C}$ seems to be caused by antioxidant activity [22], by reducing the enzymatically generated $o$-quinones [23] and by inhibiting tyrosinasa activity as shown by one recent paper published by Choi et al. [31], where they treated 20 patients with melasma with hemiface iontophoresis using topical Vitamin C and under Western blot analysis, they showed that Vitamin C inhibited the expression of tyrosinase and therefore acted as inhibitor of melanogenesis [31].

Several studies demonstrated that skin viscoelasticity values obtained by cutometer, which indicate the biomechanical properties of the skin, decreases with age [32-34]. Furthermore, photo aged dermis contains significantly reduced levels of collagen types I and III, fewer anchoring fibrils at the DermalEpidermal Junction (DEJ; collagen VII) and loss of the fibrillin-rich microfibrillar architecture in the papillary dermis [35]. These histological aspects of photoaging are known to be reverted by the action of the three ingredients present on the topical product of the study. A concordance between the improvement of skin elasticity and firmness as well as the improvement of the depth of skin wrinkles exist. These three signs can be explained by the dermal preservation after UV radiation, the stimulating repair of dermal components and the dermal synthesis induction promoted by the combination of these topical active components.

During the present study, a significant increase of firmness and elasticity on Asian and Caucasian women was detected. The improvement was significantly achieved within the first 15 days and higher and also significant after one month of treatment. This action could be explained by the stimulation of collagen and elastic fibers induced by protecoglycans, by the action of Vitamin C, that directly stimulates the synthesis of collagen I and III and inhibits the degradation of the extracellular matrix and mainly by the SCA activity on the stimulation of fibroblasts and increasing the content on collagen and elastic fibers.

The cosmetic awareness is constantly increasing and a higher demand for a complete cosmetic product exists. The proper cosmetic product can be used in monotherapy or as home continuing care that would enhance the results of the cosmetic techniques performed at the clinic. A great number of topical antiaging formulations containing different natural extracts or vitamins have been claimed for their protective and rejuvenating effects. The present study was performed over 40 patients (20 Caucasian and 20 Asian women) and we demonstrated the synergic effect obtained with the combination of three ingredients that reinforced the action mechanism of each one and results in an objective improvement of the clinical signs of photoaging as soon as after 15 days of treatment.

The significant reduction of wrinkles observed would be explained by the induction on dermal components synthesis of SCA, Vitamin C and proteoglycans, and the inhibition of dermal degradation by SCA and Vitamin C and its photo protective effect. SCA promoted fibroblast survival and proliferation and down regulates Matrix Metalloproteinase expression in dermal fibroblasts. Brieva et al. [36] demonstrated that SCA significantly inhibited MMP-1 and MMP-2 expression, suggesting that limitation of MMP expression contributes to its regenerative properties. The clinical studies performed with SCA, as only treatment, demonstrated that this ingredient is able to increases skin thickness and reduces the elastosis, as showed the histological study, and reduces roughness and wrinkle depth, after 3 month of treatment [12,13]. Even more, SCA demonstrated to improve the clinical signs of aging on the neck and chest [37]. 


\section{Conclusions}

In conclusion, this study provides evidence that a product formulated by a combination of Crytomphalus aspersa secretion (SCA), proteoglycans and Vitamin $\mathrm{C}$, is able to induce clinically identifiable improvement in the appearance of facial wrinkles after two weeks of use. Following 30 days, all analyzed parameters presented a constant improvement, statistically significance. This improvement is in correlation with the improvement of objective signs of photoaging such as elasticity, firmness, luminosity and skin moisturization. The study supports the use of this combination twice a day at least by one month to reach an effective photoaging repair and therefore, justifies it use as intensive treatment.

\section{Acknowledgement}

The authors acknowledge Cantabria labs (Industrial Farmaceutica Cantabria, SA) for the partial contribution in financing the clinical trial and the study's product (Endocare ${ }^{\circledR} \mathrm{C}$ proteoglicanos oil free/Endocare ${ }^{\circledR} \mathrm{C}$ Pure concentrate) support.

\section{References}

1. Zouboulis CC, Makrantonaki E (2011) Clinical aspects and molecular diagnostics of skin aging. Clin Dermatol 29: 3-14.

2. Tigges J, Krutmann J, Fritsche E, Haendeler J, Schaal H, et al. (2014) The hallmarks of fibroblast ageing. Mech Ageing Dev 138: 26-44.

3. Pittayapruek $P$, Meephansan J, Prapapan $O$, Komine $M$, Ohtsuki M (2016) Role of Matrix Metalloproteinases in Photoaging and Photocarcinogenesis. Int J Mol Sci 17: E868.

4. Quan T, Little E, Quan H, Qin Z, Voorhees JJ, et al. (2013) Elevated matrix metalloproteinases and collagen fragmentation in photodamaged human skin: Impact of altered extracellular matrix microenvironment on dermal fibroblast function. J Invest Dermatol 133: 1362-1366.

5. Quan T, Fisher GJ (2015) Role of Age-Associated Alterations of the Dermal Extracellular Matrix Microenvironment in Human Skin Aging: A Mini-Review. Gerontology 61: 427-434.

6. Ganceviciene R, Liakou A, Theodoridis A, Makrantonaki E, Zouboulis CC (2012) Skin anti-aging strategies. Dermatoendocrinol 4: 308-319.

7. Draelos ZD (2017) The Role of a Natural Mollusk Egg-Derived Ingredient in Facial Appearance. J Drugs Dermatol 16: 678-681.

8. Fabi SG, Massaki A, Eimpunth S, Pogoda J, Goldman MP (2013) Evaluation of microfocused ultrasound with visualization for lifting, tightening, and wrinkle reduction of the décolletage. J Am Acad Dermatol 69: 965-971.

9. Gonzalez S, Juarranz A, Reyes E, Matabuena M, Lucena S (2014) Extract from Cryptomphalus aspersa eggs significantly promotes skin homeostasis and migration and survival of skin cells in vitro. J Am Acad Dermatol 70: AB17.

10. Espada J, Salazar N, Damian A, Cifuentes A, Garcia-Honduvilla $\mathrm{N}$, et al. (2014) Cryptomphalus aspersa mollusk eggs extract promotes migration and regenerative behavior of human keratinocytes and mesenchymal stem cells in vitro. J Investig Dermatol 134: S4.
11. Cruz MC, Sanz-Rodríguez F, Zamarron A, Reyes E, Carrasco E, et al. (2012) A secretion of the mollusk Cryptomphalus aspersa promotes proliferation, migration and survival of keratinocytes and dermal fibroblasts in vitro. Int J Cosmet Sci 34: 183-189.

12. Tribo-Boixareu MJ, Parrado-Romero C, Rais B, Reyes E, Vitale M, et al. (2009) Clinical and Histological Efficacy of a Secretion of the Mollusk Cryptomphalus aspersa in the Treatment of Cutaneous Photoaging. J Cosmet Dermatol 22: 247-252.

13. Fabi SG, Cohen JL, Peterson JD, Kiripolsky MG, Goldman MP (2013) The Effects of Filtrate of the Secretion of the Cryptomphalus aspersa on Photoaged Skin. J Drugs Dermatol 12: 453-457.

14. Ghatak S, Maytin EV, Mack JA, Hascall VC, Atanelishvili I, et al. (2015) Roles of Proteoglycans and Glycosaminoglycans in Wound Healing and Fibrosis. Int J Cell Biol 2015: 834893.

15. Carrino DA, Onnerfjord P, Sandy JD, Cs-Szabo G, Scott PG, et al. (2003) Age-related changes in the proteoglycans of human skin. Specific cleavage of decorin to yield a major catabolic fragment in adult skin. J Biol Chem 278: 17566-17572.

16. Shin JE, Oh JH, Kim YK, Jung JY, Chung JH (2011) Transcriptional Regulation of Proteoglycans and Glycosaminoglycan Chainsynthesizing Glycosyltransferases by UV Irradiation in Cultured Human Dermal Fibroblasts. J Korean Med Sci 26: 417-424.

17. Reed CC, lozzo RV (2002) The role of decorin in collagen fibrillogenesis and skin homeostasis. Glycoconj J 19: 249-255.

18. Corsi A, Xu T, Chen XD, Boyde A, Liang J, et al. (2002) Phenotypic effects of biglycan deficiency are linked to collagen fibril abnormalities, are synergized by decorin deficiency, and mimic Ehlers-Danlos-like changes in bone and other connective tissues. J Bone Miner Res 17: 1180-1189.

19. Reinboth B, Hanssen E, Cleary EG, Gibson MA (2002) Molecular interactions of biglycan and decorin with elastic fiber components biglycan forms a ternary complex with tropoelastin and microfibril-associated glycoprotein 1. J Biol Chem 277: 3950-3957.

20. Espinal-Perez LE, Moncada B, Castanedo-Cazares JP (2004) A double-blind randomized trial of $5 \%$ ascorbic acid vs. $4 \%$ hydroquinone in melisma. Int J Dermatol 43: 604-607.

21. Catani MV, Rossi A, Costanzo A, Sabatini S, Livrero M, et al. (2001) Induction of gene expression via activator protein-1 in the ascorbate protection against UV-induced damage. Biochem J 356: 77-85.

22. Boyera N, Galey I, Bernard BA (1998) Effect of vitamin C and its derivatives on collagen synthesis and cross-linking by normal human fibroblasts. Int J Cosmet Sci 20: 151-158.

23. Kameyama K, Sakai C, Kondoh S, Yonemoto K, Nishiyama S, et al. (1996) Inhibitory effect of magnesium 1-ascorbyl-2-phosphate (VC-PMG) on melanogenesis in vitro and in vivo. J Am Acad Dermatol 34: 29-33.

24. Hanson KM, Simon JD (1998) Epidermal trans-uronic acid and the UV-A- induced photoaging of the skin. Proc Natl Acad Sci U S A 95: 10576-10578.

25. Darr D, Combs S, Dunston S, Manning T, Pinnell S (1992) Topical vitamin $C$ protects porcine skin from ultraviolet radiationinduced damage. Br J Dermatol 127: 247-253. 
26. Perricone $N$ (1993) The photoprotective and anti-inflammatory effects of topical ascorbyl palmitate. J Geriatr Dermatol 1: 3-10.

27. Dreher F, Maibach H (2001) Protective effects of topical antioxidants in humans. Curr Probl Dermatol 29: 157-164.

28. Fitzpatrick R, Rostan E (2002) Double-blind, half-face study comparing topical vitamin $C$ and vehicle for rejuvenation of photodamage. Dermatol Surg 28: 231-236.

29. Farris PK (2005) Topical vitamin C: a useful agent for treating photoaging and other dermatologic conditions. Dermatol Surg 31: 814-817.

30. Crisan D, Roman I, Crisan M, Scharffetter-Kochanek K, Badea R (2015) The role of vitamin $C$ in pushing back the boundaries of skin aging: an ultrasonographic approach. Clin Cosmet Investig Dermatol 8: 463-470.

31. Choi YK, Rho YK, Yoo KH, Lim YY, Li K, et al. (2010) Effects of vitamin $C$ vs. multivitamin on melanogenesis: Comparative study in vitro and in vivo. Int J Dermatol 49: 218-226.

32. Longo C, Ciardo S, Pellacani G (2015) Non-invasive, investigative methods in skin aging. G Ital Dermatol Venereol 150: 675-686.
33. Mercurio DG, Jdid R, Morizot F, Masson P, Maia Campos PM (2016) Morphological, structural and biophysical properties of French and Brazilian photoaged skin. Br J Dermatol 174: 553561.

34. Pierard GE, Hermanns-Lê T, Gaspard U, Piérard-Franchimont C (2014) Asymmetric facial skin viscoelasticity during climacteric aging. Clin Cosmet Investig Dermatol 7: 111-118.

35. Watso REB, Ogden S, Cotterell LF, Bowden JJ, Bastrilles JY, et al. (2009) A cosmetic 'anti-ageing' product improves photoaged skin: a double-blind, randomized controlled trial. Br J Dermatol 161: 419-426.

36. Brieva A, Philips N, Tejedor R, Guerrero A, Pivel JP, et al. (2008) Molecular Basis for the Regenerative Properties of a Secretion of the Mollusk Cryptomphalus aspersa. Skin Pharmacol Physiol 21: 15-22.

37. Trink A, Rinaldi F (2013) Double-blind pilot study on efficacy and tolerabilty of a topical formulation containing SCA in the treatment of photoaging. Hitech dermo 45-49. 